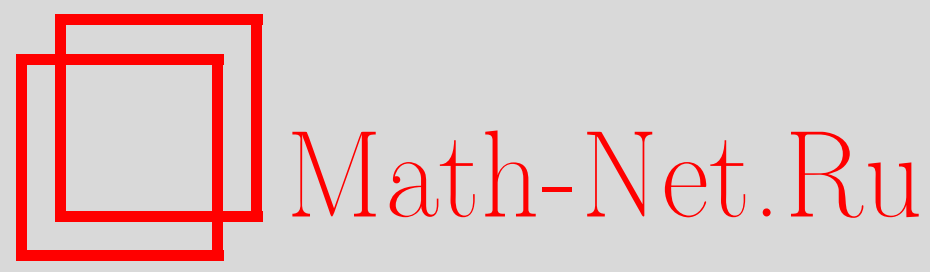

М. В. Волков, И. А. Гольдберг, Тождества полугрупп треугольных матриц над конечными полями, Матем. заметкu, 2003, том 73, выпуск 4, 502-510

DOI: https://doi.org/10.4213/mzm210

Использование Общероссийского математического портала Math-Net.Ru подразумевает, что вы прочитали и согласны с пользовательским соглашением http://www.mathnet.ru/rus/agreement

Параметры загрузки:

IP: 54.162 .127 .20

26 апреля 2023 г., $16: 17: 59$

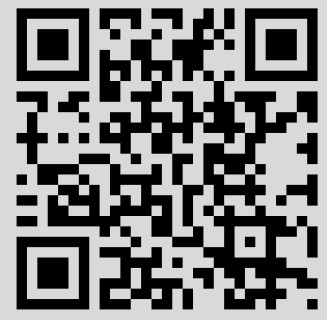




\title{
ТОЖДЕСТВА ПОЛУГРУПП ТРЕУГОЛЬНЫХ МАТРИЦ НАД КОНЕЧНЫМИ ПОЛЯМИ
}

\author{
М.В. Волков, И. А. Гольдберг
}

\begin{abstract}
Доказывается, что полугруппа всех треугольных $(n \times n)$-матриц над конечньм полем $K$ существенно бесконечно базируема тогда и только тогда, когда $n>3$ и $|K|>2$.

Библиографоия: 10 названий.
\end{abstract}

В то время как тождества любой конечной групшы обладают конечным базисом [1], среди конечных полугрупп имеются и не конечно базируемые- первым примером такого рода была 6 -элементная полугруппа $B_{2}^{1}$, образованная $(2 \times 2)$-матрицами

$$
\left(\begin{array}{ll}
0 & 0 \\
0 & 0
\end{array}\right), \quad\left(\begin{array}{ll}
1 & 0 \\
0 & 1
\end{array}\right), \quad\left(\begin{array}{ll}
1 & 0 \\
0 & 0
\end{array}\right), \quad\left(\begin{array}{ll}
0 & 1 \\
0 & 0
\end{array}\right), \quad\left(\begin{array}{ll}
0 & 0 \\
1 & 0
\end{array}\right), \quad\left(\begin{array}{ll}
0 & 0 \\
0 & 1
\end{array}\right)
$$

относительно обычного матричного умножения [2]. Естественно возникает задача классификации конечных полугрупп с точки зрения конечной базируемости их тождеств. За последние 30 лет в этом направлении проделана большая работа, результаты которой за первую и вторую половину указанного периода систематизированы соответственно в [3] и [4]. Представляется, однако, что эта работа еще далека от полного завершения. В частности, для ряда конкретных конечных полугрупп, важных для теории и приложений, все еще неизвестно, конечен ли их базис тождеств. Среди “неподдающихся" были до настоящего времени и полугрупшы треугольных матриц над конечными полями, вопрос о конечной базируемости которых был в явном виде поставлен еще в [3] и недавно повторен в [4]. В данной заметке мы показьваем, что тождества всякой полугрупш треугольных матриц размера не менее чем $4 \times 4$ над конечным полем порядка не менее чем 3 не обладают конечным базисом.

В действительности, получен гораздо более сильньй результат, для формулировки которого понадобится одно понятие из теории локально конечных многообразий. (Напомним, что многообразие $\mathbf{V}$ локально конечно, если каждая конечно порожденная полугруппа из $\mathbf{V}$ конечна.) Локально конечное многообразие $\mathbf{V}$ назьвается существенно бесконечно базируемым, если любое локально конечноемногообразие, содержащее $\mathbf{V}$, не имеет конечного базиса тождеств. Разумеется, при этом и само многообразие $\mathbf{V}$ не конечно базируемо.

Наименьшее многообразие, содержащее данную полугруппу $S$, назовем многообразием, порожденным $S$, и обозначим через $\operatorname{var} S$. Хорошо известно, что для конечной 
полугруппы $S$ многообразие var $S$ локально конечно [5, теорема 14.4]. Благодаря этому, можно распространить интересующее нас понятие на полугруппы: конечная полугруппа $S$ существенно бесконечно базируема, если таково многообразие var $S$. Целью настоящей заметки является доказательство следующего результата.

Теорема. Полугруппа $T_{n}(K)$ всех верхнетреугольных $(n \times n)$-матрии над конечным полем $K$ существенно бесконечно базируема тогда и только тогда, когда $|K|>2$ u $n>3$.

Самым известным (и исторически первым) примером существенно бесконечно базируемой полугруппы является упомянутая в начале введения 6-элементная полугруппа $B_{2}^{1}$, см. [6, следствие 6.1]. Понятно, что и всякая конечная полугруппа $S$ такая, что $B_{2}^{1} \in \operatorname{var} S$, будет существенно бесконечно базируемой. Именно так - с помощью отыскания полугрупшы $B_{2}^{1}$ в соответствуюшем многообразии - доказьвалась до сих пор существенная бесконечная базируемость важных конкретных полугрупп, например, полугруппы всех преобразований $n$-элементного множества (при $n>2$ ) и полугрупш всех $(n \times n)$-матриц над конечным полем (при $n>1)$ [6, следствие 6.2]. Отметим в этой связи, что полугруппа $B_{2}^{1}$ не принадлежит многообразию $\operatorname{var} T_{n}(K)$ ни при каких $n$ и $K$ (см. лемму 3 и следствие 2 ниже), и потому наша теорема не может быть получена указанным методом. Можно сказать, что полугруппы верхнетреугольных матриц над конечными полями составляют первьй класс примеров существенно бесконечно базируемых полугрупп “естественного происхождения", не сводящихся к $B_{2}^{1}$.

Совокупность всех верхнетреугольных $(n \times n)$-матриц над полем является не только полугруппой, но и кольцом. В [6, следствие 6.3] доказано, что если мультипликативная полугрупша конечного кольца $R$ имеет конечньй базис тождеств, то факторкольцо кольца $R$ по его радикалу - прямая сумма конечных полей. Мы видим теперь, что это утверждение нельзя обратить: факторкольцо кольца $T_{n}(K)$ по его радикалу - прямая сумма $n$ копий поля $K$, а полугруппа $T_{n}(K)$ при $|K|>2$ и $n>3$ не конечно базируема. Насколько нам известно, в литературе такие примеры ранее не отмечались.

Достаточность условия теоремы будет доказана в п. 2, необходимость - в п. 3. В подготовительном п. 1 собраны необходимые сведения о полугруппах $T_{n}(K)$ и о существенно бесконечно базируемых конечных полугруппах.

Используемые в работе понятия теории полугрупп (в частности, определения эквивалентностей Грина $\mathscr{D}, \mathscr{R}$ и $\mathscr{L})$ можно найти в книге [7].

1. Предварительные сведения. Пусть $a$ и $b$-элементы полугрупшы $S$ с единищей. Скажем, что $a$ делит $b$, если $b=x a y$ для некоторых $x, y \in S$. Для матрицы $a=\left(\alpha_{i j}\right)$ из $T_{n}(K)$ положим $\Delta(a)=\left\{i \mid \alpha_{i i} \neq 0\right\}$. Следующее свойство идемпотентных верхнетреугольных матриц (над произвольньп полем) по всей вероятности известно, но для полноты мы приводим его с доказательством.

ЛЕмма 1. Если е - идемпотентная матрица из $T_{n}(K)$, то $а \in T_{n}(K)$ делите тогда и только тогда, когда $\Delta(e) \subseteq \Delta(a)$.

ДокАЗАТЕЛЬСтво. Из равенства $e=x a y$, где $e=\left(\varepsilon_{i j}\right), a=\left(\alpha_{i j}\right), x, y \in T_{n}(K)$, следует, что если $\alpha_{i i}=0$ для некоторого $i$, то и $\varepsilon_{i i}=0$. Поэтому если $a$ делит $e$, то $\Delta(e) \subseteq \Delta(a)$. Обратно, пусть $\Delta(e) \subseteq \Delta(a)$. Возьмем произвольную матрицу $b=\left(\beta_{i j}\right) \in$ 
$T_{n}(K)$, диагональные элементы которой подчинены равенствам

$$
\beta_{i i}= \begin{cases}\alpha_{i i}^{-1}, & \text { если } i \in \Delta(e), \\ 0, & \text { если } i \notin \Delta(e) .\end{cases}
$$

Тогда $\alpha_{i i} \beta_{i i}=\varepsilon_{i i}$ для всех $i=1, \ldots, n$, т.е. главные диагонали матриц $е$ и $a b$ одинаковы. Следовательно, матрица $e-a b$ имеет нулевую главную диагональ, откуда $(e-a b)^{n}=0$. Раскрыв скобки и приведя подобные, можно переписать это равенство в виде $e-x a y=0$ для некоторых матриц $x, y \in T_{n}(K)$. Таким образом, $a$ делит $e$.

В дальнейшем всегда будем считать поле $K$ конечным; тогда, разумеется, и полугруппа $T_{n}(K)$ конечна. В конечной полугруппе с единицей два элемента делят друг друга тогда и только тогда, когда они находятся в одном классе эквивалентности Грина $\mathscr{D}$, см. $[7, \S 6.6]$. Поэтому из леммы 1 немедленно вытекает

СледСтвИЕ 1. Две идемпотентные матрицы из $T_{n}(K)$ принадлежсат одному D-классу этой полугруппы тогда и только тогда, когда их главные диагонали одинаковы.

Если $e$ - идемпотент некоторой полугрупшы $S$, то через $H_{e}$ обозначают максимальную подгруппу в $S$, единицей которой служит $e$. Нам понадобится информация о порядках максимальных подгрупп полугруппы $T_{n}(K)$.

Лемма 2. Пусть е-идемпотент из $T_{n}(K), \delta$ - число әлементов в множестве $\Delta\left(\right.$ e). Тогда порядок подгруппы $H_{е}$ равен $(|K|-1)^{\delta}|K|^{\delta(\delta-1) / 2}$.

ДокАЗАТЕЛьСтво. Максимальные подгруппы, единицы которых $\mathscr{D}$-эквивалентны, изоморфны [7, теорема 2.20]. Поэтому согласно следствию 1 можно заменить матрицу $e$ ее главной диагональю; иначе говоря, можно считать, что $e$ - диагональная матрица. Покажем, что тогда подгруппа $H_{e}$ состоит из всех таких верхнетреугольных матриц $a=\left(\alpha_{i j}\right)$, что

1) $\alpha_{j k}=0$, если либо $j \notin \Delta(e)$, либо $k \notin \Delta(e)$;

2) $\alpha_{i i} \neq 0$, если $i \in \Delta(e)$.

Из этого с очевидностью будет следовать утверждение леммы. В самом деле, число матриц, удовлетворяющих 1) и 2), как раз равно $(|K|-1)^{\delta}|K|^{\delta(\delta-1) / 2}$ : каждьй из $\delta$ элементов $\alpha_{i i}$, где $i \in \Delta(e)$, может принимать любое значение из множества $K \backslash\{0\}$, а каждьй из $\delta(\delta-1) / 2$ элементов $\alpha_{j k}$, где $j, k \in \Delta(e)$ и $j<k$, может принимать любое значение из $K$.

Матрицы $a$, принадлежащие подгрупе $H_{e}$, характеризуются тем, что $a e=e a=a$ и $a^{\ell}=e$ для некоторого натурального $\ell$. Легко понять, что указанные равенства влекут выполнение условий 1$)$ и 2) соответственно. Обратно, если матрища $a \in T_{n}(K)$ удовлетворяет 1), то $a e=e a=a$. Остается проверить, что при вьполнении 1) и 2) $a^{\ell}=e$ для подходящего $\ell$. Будем доказьвать это индукцией по размеру матрицы.

Ясно, что проверяемое утверждение справедливо для $(1 \times 1)$-матриц. Далее, если $e$ - единичная матрица, то в силу 2) $a$ - обратимая матрица, откуда $a^{\ell}=e$, где $\ell=(|K|-1)^{n}|K|^{n(n-1) / 2}$-порядок группы обратимых верхнетреугольных $(n \times n)$-матриц. Поэтому будем считать, что $n>1$ и что нуль встречается на главной диагонали 
матрицы $e$. Тогда эту матрицу можно представить в виде

$$
e=\left(\begin{array}{lll}
e_{1} & & \\
& 0 & \\
& & e_{2}
\end{array}\right) \text {, }
$$

где $e_{1}$ и $e_{2}$ - диагональные матрицы меньших размеров (одна из которых может отсутствовать). Согласно 1) соответствующим образом должна быть устроена и матрица $a$, а именно,

$$
a=\left(\begin{array}{ccc}
a_{1} & o_{1} & b \\
& 0 & o_{2} \\
& & a_{2}
\end{array}\right),
$$

где $a_{1}$ и $a_{2}$ - такие верхнетреугольные матрицы, что $a_{i} e_{i}=e_{i} a_{i}=a_{i}(i=1,2), o_{1}\left[o_{2}\right]-$ нулевая колонка [строка], а $b$-прямоугольная матрица со свойством $e_{1} b=b=b e_{2}$. По предположению индукции найдутся такие показатели $\ell_{1}$ и $\ell_{2}$, что $a_{i}^{\ell_{i}}=e_{i}(i=1,2)$. Если $m$ - наименьшее общее кратное этих показателей, то

$$
a^{m}=\left(\begin{array}{ccc}
e_{1} & o_{1} & c \\
& 0 & o_{2} \\
& & e_{2}
\end{array}\right)
$$

где $e_{1} c=c=c e_{2}$, и для любого натурального $k$

$$
a^{m k}=\left(\begin{array}{ccc}
e_{1} & o_{1} & k c \\
& 0 & o_{2} \\
& & e_{2}
\end{array}\right) .
$$

Отсюда, выбирая в качестве $k$ характеристику $p$ поля $K$, получаем $a^{m p}=e$, что и требовалось.

Через DS обозначается класс конечных полугрупп, в которых каждьй $\mathscr{D}$-класс, содержаший идемпотент, является подполугруппй. Наша очередная цель - показать, что полугруппы $T_{n}(K)$ принадлежат этому классу. Воспользуемся следующей леммой, в которой для случая полугруп с единищей специализированы некоторые из характеризаций класса DS, найденных в [8, теорема 3$]$ и [9, лемма 1].

Лемма 3. Для конечной полугруппы $S$ с единицей следующие условия әквивалентны:

(a) $S \in \mathbf{D S}$;

(б) полугруппа $B_{2}^{1}$ не принадлежст многообразию $\operatorname{var} S$;

(в) если каждый из әлементов $a, b \in S$ делит идемпотент $e \in S$, то и произведение $a b$ делит е;

(г) если әлемент $a \in S$ делит идемпотент $е \in S$, то произведение еае принадлежит максимальной подгруппе $H_{e}$.

СлеДСТВИЕ 2. Для любых $n$ u $K$ nолугруппа $T_{n}(K)$ nринадлежстт DS. 
ДокАЗАТЕЛЬСтво. Проверим, что полугруппа $T_{n}(K)$ удовлетворяет условию (в) леммы 3. Пусть $e$ - идемпотентная матрица из $T_{n}(K)$ и $a, b \in T_{n}(K)$ делят $e$. Тогда по лемме $1 \Delta(e) \subseteq \Delta(a)$ и $\Delta(e) \subseteq \Delta(b)$, откуда $\Delta(e) \subseteq \Delta(a) \cap \Delta(b)=\Delta(a b)$. Снова применяя лемму 1 , заключаем, что произведение $a b$ делит $e$.

Из леммы 3 и следствия 2 вытекает, в частности, упомянутьй во введении факт, что полугруппа $B_{2}^{1}$ не принадлежит многообразию $\operatorname{var} T_{n}(K)$ ни при каких $n$ и $K$.

Нам понадобится критерий существенной бесконечной базируемости из [9]. Перед тем, как сформулировать его, напомним, что период $d(S)$ конечной полугруппы $S$ - это наименьшее общее кратное периодов ее подгрупп, а верхним гиперцентром $\Gamma(G)$ конечной группы $G$ назьвается подгрупп, на которой стабилизируется верхний центральньй ряд $G$.

ПРЕДЛОЖЕНИЕ 1. Конечная полугруппа $S$ с единиией существенно бесконечно базируема тогда и только тогда, когда найдутся әлемент $a \in S$ и идемпотент e $\in S$ такие, что а делит е и әлементы еае $и$ еа $a^{d(S)+1}$ е не принадлежст одному смехному классу подгруппы $H_{e}$ по ее верхнему гипериентру.

Если элементы $a, e \in S$ обладают свойством из формулировки предложения 1 , то будем называть пару $(a, e)$ критической. Отметим, что бьвают ситуации, когда это свойство удовлетворяется, так сказать, тривиальным образом просто в виду того, что элементы $е a е$ и $e a^{d(S)+1} e$ не попадают в подгруппу $H_{e}$. Именно так, например, обстоит дело для полугрушшы $B_{2}^{1}$, в которой критической является пара матриц

$$
a=\left(\begin{array}{ll}
0 & 1 \\
0 & 0
\end{array}\right), \quad e=\left(\begin{array}{ll}
1 & 0 \\
0 & 0
\end{array}\right) .
$$

(Действительно, $d\left(B_{2}^{1}\right)=1$ и еae $=e a^{2} e=0 \notin H_{e}=\{e\}$. ) Однако для полугрупп $T_{n}(K)$ из условий (в) и (г) леммы 3 легко вывести, что если некоторая матрица $a \in T_{n}(K)$ делит идемпотент $e \in T_{n}(K)$, то $e a^{k} e \in H_{e}$ для всех $k$, а стало быть, существенная бесконечная базируемость, если она имеется, должна проявляться нетривиальным образом.

В п. 3 будет полезен следующий факт, полученный в [10].

ПРЕДЛОЖЕНИЕ 2. Пусть $S$ - такая существенно бесконечно базируемая полугруппа, что $B_{2}^{1} \notin \operatorname{var} S, u(a, e)$ - произвольная критическая пара в $S$. Тогда $\mathscr{D}$-класс идемпотента е содержит по крайней мере три $\mathscr{L}$-класса и по крайней мере три $\mathscr{R}$-класса.

2. Достаточность. Покажем, что полугруппы $T_{n}(K)$ при $n \geqslant 4$ и $|K|>2$ являются существенно бесконечно базируемыми. Положим

$$
e=e_{11}+e_{12}+e_{34}+e_{44}, \quad a=e_{11}+e_{23}+e_{44}
$$

где $e_{i j}$, как обычно, обозначает матричную единииу, т.е. матрицу, у которой на месте $(i, j)$ стоит 1 , а все остальные элементы равны нулю. Существенная бесконечная базируемость полугруппы $T_{n}(K)$ вытекает из предложения 1 и из следующего факта.

ПРЕДЛОЖЕНИЕ 3. Матрицы а и е образуют критическую пару в $T_{n}(K)$. 
ДокАЗАТЕльство. С учетом известного правила умножения матричных единиц:

$$
e_{i j} e_{k \ell}= \begin{cases}e_{i \ell}, & \text { если } j=k, \\ 0, & \text { если } j \neq k,\end{cases}
$$

легко подсчитать, что $e$ - идемпотент. Отсюда в силу леммы 1 вытекает, что $a$ делит $e$. Так же непосредственно можно убедиться, что $a^{2}=a^{3}=e_{11}+e_{44}$. Отсюда $a^{k}=e_{11}+e_{44}$ и $e a^{k} e=e$ для любого $k>1$. В частности, если $d-$ период полугруппы $T_{n}(K)$, то матрица $e a^{d+1} e=e$ принадлежит верхнему гиперцентру $\Gamma\left(H_{e}\right)$ подгруппы $H_{e}$. Итак, для того, чтобы доказать, что элементы $e a e$ и $e a^{d+1} e$ не принадлежат одному смежному классу по $\Gamma\left(H_{e}\right)$, остается проверить, что

$$
e a e=e_{11}+e_{12}+e_{14}+e_{34}+e_{44} \notin \Gamma\left(H_{e}\right) .
$$

Пусть $\{e\}=Z_{0} \subset Z_{1} \subset Z_{2} \subset \cdots \subset Z_{m}=\Gamma\left(H_{e}\right)$ - верхний центральный ряд группы $H_{e}$. Если $g \in \Gamma\left(H_{e}\right), h \in H_{e}$, то коммутатор $[g, h]$ принадлежит уже $Z_{m-1}$, повторньй коммутатор $[[g, h], h]$ попадает в $Z_{m-2}$, и т.д.; наконец, $m$-кратное коммутирование с $h$ дает $e$. Поэтому для того, чтобы показать, что еae $\notin \Gamma\left(H_{e}\right)$, достаточно найти такую матрицу $h \in H_{e}$, что

$$
[\ldots[[e a e, \underbrace{h], h], \ldots, h}_{m \text { раз }}] \neq e
$$

ни при каком натуральном $m$. Пусть $\beta$ - элемент поля $K$, отличный от 0 и 1 (такой элемент существует, так как $|K|>2$ ). Убедимся, что в качестве $h$ можно взять матрицу $\beta^{-1}\left(e_{11}+e_{12}\right)+e_{34}+e_{44}$. Прежде всего, нетрудно проверить, что $h \in H_{e}$ и что обратный к $h$ элемент из $H_{e}$ есть $\beta\left(e_{11}+e_{12}\right)+e_{34}+e_{44}$. Далее, для произвольного $\gamma \in K$ рассмотрим матрицу

$$
g_{\gamma}=e_{11}+e_{12}+\gamma e_{14}+e_{34}+e_{44}
$$

Она также принадлежит подгрупше $H_{e}$, причем обратньй к $g_{\gamma}$ элемент этой подгруппы равен $e_{11}+e_{12}-\gamma e_{14}+e_{34}+e_{44}$. Вычислим коммутатор $\left[g_{\gamma}, h\right]$ :

$$
\begin{aligned}
{\left[g_{\gamma}, h\right]=} & \left(e_{11}+e_{12}-\gamma e_{14}+e_{34}+e_{44}\right) \cdot\left(\beta\left(e_{11}+e_{12}\right)+e_{34}+e_{44}\right) \\
& \times\left(e_{11}+e_{12}+\gamma e_{14}+e_{34}+e_{44}\right) \cdot\left(\beta^{-1}\left(e_{11}+e_{12}\right)+e_{34}+e_{44}\right) \\
= & \left(\beta\left(e_{11}+e_{12}\right)-\gamma e_{14}+e_{34}+e_{44}\right) \times\left(\beta^{-1}\left(e_{11}+e_{12}\right)+\gamma e_{14}+e_{34}+e_{44}\right) \\
= & e_{11}+e_{12}+\gamma(\beta-1) e_{14}+e_{34}+e_{44}=g_{\gamma(\beta-1)} .
\end{aligned}
$$

Отсюда, замечая, что еае $=g_{1}$, получаем, что при любом натуральном $m$

$$
\left[\ldots \left[[e a e, \underbrace{h], h], \ldots, h}_{m \text { раз }}]=g_{(\beta-1)^{m}} \neq g_{0}=e,\right.\right.
$$

что и требовалось. 
3. Необходимость. Будем назьвать конечную полугрупп слабо конечно базируемой, если она не является существенно бесконечно базируемой, т.е. если она содержится в некотором конечно базируемом локально конечном многообразии. Нам нужно доказать, что полугруппа $T_{n}(K)$ слабо конечно базируема, если $|K|=2$ или $n \leqslant 3$.

Если $|K|=2$, то по лемме 2 порядок любой подгрупшы в $T_{n}(K)$ есть степень двойки. Хорошо известно, что любая группа такого порядка нильпотентна. Как отмечено в [9, теорема 2], если все подгруппы конечной полугруппы $S$ нильпотентны и $B_{2}^{1} \notin \operatorname{var} S$, то $S$ слабо конечно базируема. Поскольку $B_{2}^{1} \notin \operatorname{var} T_{n}(K)$ при любых $n$ и $K$ (см. следствие 2 и лемму 3), указанньй признак слабой конечной базируемости приложим к полугруппам верхнетреугольных матриц над двухэлементным полем.

Пусть теперь поле $K$ произвольно, но $n \leqslant 3$. Ясно, что свойство “быть слабо конечно базируемой" наследуется подполугрупшами, поэтому если мы покажем, что данньп свойством обладает полугруппа $T_{3}(K)$, то отсюда будет следовать и слабая конечная базируемость полугрупп $T_{1}(K)$ и $T_{2}(K)$, которые очевидньм образом вкладываются в $T_{3}(K)$. (В действительности, полугруппа $T_{1}(K)$ - т.е. мультипликативная полугруппа поля $K$ - даже конечно базируема, поскольку она коммутативна [2, теорема 9].)

Итак, пусть $e=\left(\varepsilon_{i j}\right)$ - произвольньй идемпотент полугруппы $T_{3}(K)$. В силу предложения 1 достаточно проверить, что $e$ не образует критическую пару ни с одной матрицей из $T_{3}(K)$. В зависимости от распределения нулей и единиц на главной диагонали матрицы $е$ возможны 4 случая, которые мы последовательно рассмотрим.

Случай 1: $\varepsilon_{11}=\varepsilon_{33}=1$. Покажем, что в этом случае любая матрица $a=\left(\alpha_{i j}\right)$, делящая $e$, принадлежит некоторой подгрупе полугрупш $T_{3}(K)$. Так как период любой такой подгруппы делит число $d=d\left(T_{3}(K)\right)$, отсюда будет следовать, что $a^{d+1}=a$. Поэтому $е a^{d+1} e=e a е$ и, поскольку по следствию 2 и лемме 3 матрицы $e a^{d+1}$ е и еае принадлежат подгруппе $H_{e}$, они заведомо лежат в одном смежном классе этой подгруппы по ее верхнему гиперцентру. Следовательно, никакая пара $(a, e)$ не будет критической, что и требуется.

Итак, пусть $a$ - произвольный делитель идемпотента $e$. Тогда по лемме $1 \alpha_{11} \neq 0$ и $\alpha_{33} \neq 0$. Если к тому же и $\alpha_{22} \neq 0$, то матрища $a$ принадлежит подгруппе обратимых верхнетреугольных матриц. Поэтому будем считать, что $\alpha_{22}=0$. Вычисляя последовательные степени матрищы $a$, несложно убедиться, что

$$
a^{n}=\alpha_{11}^{n} e_{11}+\alpha_{11}^{n-1} \alpha_{12} e_{12}+\beta_{n} e_{13}+\alpha_{23} \alpha_{33}^{n-1} e_{23}+\alpha_{33}^{n} e_{33},
$$

где $\beta_{n}$ есть некий многочлен от $\alpha_{11}, \ldots, \alpha_{33}$. В конечной полугрупше некоторая степень любого элемента является идемпотентом. Если $n$ таково, что $a^{n}$ - идемпотент, то из (1) заключаем, что $\alpha_{11}^{n}=\alpha_{33}^{n}=1$, откуда $\alpha_{11}^{n-1}=\alpha_{11}^{-1}$ и $\alpha_{33}^{n-1}=\alpha_{33}^{-1}$. Поэтому для такого $n$ формулу (1) можно переписать следуюшим образом:

$$
a^{n}=e_{11}+\alpha_{11}^{-1} \alpha_{12} e_{12}+\beta_{n} e_{13}+\alpha_{23} \alpha_{33}^{-1} e_{23}+e_{33} .
$$

Возводя последнее равенство в квадрат, из условия $a^{n}=a^{2 n}$ получаем, что $\beta_{n}=$ $-\alpha_{11}^{-1} \alpha_{12} \alpha_{23} \alpha_{33}$. Таким образом,

$$
a^{n}=e_{11}+\alpha_{11}^{-1} \alpha_{12} e_{12}-\alpha_{11}^{-1} \alpha_{12} \alpha_{23} \alpha_{33}^{-1} e_{13}+\alpha_{23} \alpha_{33}^{-1} e_{23}+e_{33},
$$


откуда

$$
\begin{aligned}
a^{n+1} & =\alpha_{11} e_{11}+\alpha_{12} e_{12}+\left(-\alpha_{12} \alpha_{23} \alpha_{33}^{-1}+\alpha_{12} \alpha_{23} \alpha_{33}^{-1}+\alpha_{13}\right) e_{13}+\alpha_{23} e_{23}+\alpha_{33} e_{33} \\
& =\alpha_{11} e_{11}+\alpha_{12} e_{12}+\alpha_{13} e_{13}+\alpha_{23} e_{23}+\alpha_{33} e_{33}=a .
\end{aligned}
$$

Итак, $a^{n+1}=a$ и циклическая подполугруппа, порожденная матрицей $a$, является подгруппой.

Случай 2: $\varepsilon_{11}=1, \varepsilon_{33}=0$. Легко проверить, что $(3 \times 3)$-матрища с такой главной диагональю будет идемпотентом тогда и только тогда, когда у нее либо на месте $(2,2)$ стоит 1 , а на месте $(1,2)$ стоит 0 , либо на местах $(2,2)$ и $(2,3)$ стоят нули. В частности, идемпотент е можно представить в виде

$$
e=e_{11}+(1-\varkappa) \varepsilon_{12} e_{12}+\varepsilon_{13} e_{13}+\varkappa e_{22}+\varkappa \varepsilon_{23} e_{23}
$$

где параметр $\varkappa$ равен либо 1 , либо 0. Обозначим $\mathscr{D}$-класс идемпотента $e$ через $D$, и пусть $b$ - произвольная матрица из $D$. Поскольку в $D$ есть идемпотент, то и в каждом $\mathscr{R}$-классе, содержашемся в $D$, имеется идемпотент [7, теорема 2.11]. Пусть $f$ - некоторьй идемпотент, $\mathscr{R}$-эквивалентньй $b$. По следствию 1 у $f$ такая же главная диагональ, как у $e$, в силу чего $f$ можно представить в виде

$$
f=e_{11}+(1-\varkappa) \varphi_{12} e_{12}+\varphi_{13} e_{13}+\varkappa e_{22}+\varkappa \varphi_{23} e_{23} .
$$

Перемножая $е$ и $f$ и учитывая, что $\varkappa^{2}=\varkappa$, убеждаемся, что $e f=f$, а $f e=e$, откуда эти идемпотенты $\mathscr{R}$-эквивалентны между собой. Следовательно, матрица $b$ лежит в одном $\mathscr{R}$-классе с $e$. Итак, $\mathscr{D}$-класс идемпотента $e$ содержит лишш один $\mathscr{R}$-класс, а тогда по предложению 2 е не образует критическую пару ни с одной матрицей из $T_{3}(K)$.

Случай 3: $\varepsilon_{11}=0, \varepsilon_{33}=1$. В этом - симметричном предыдущему - случае $\mathscr{D}$-класс идемпотента $е$ содержит лишь один $\mathscr{L}$-класс и снова срабатывает предложение 2.

Случай 4: $\varepsilon_{11}=\varepsilon_{33}=0$. Покажем, что в этом случае подгруппа $H_{e}$ совпадает со своим верхним гиперцентром. Этого достаточно, чтобы утверждать, что ни для какого делителя $a$ идемпотента $е$ пара $(a, e)$ не будет критической. Действительно, как мы уже неоднократно отмечали, обе матрицы $e a^{d+1} e$ и еae принадлежат подгруппе $H_{e}$, а при условии, что $H_{e}=\Gamma\left(H_{e}\right)$, эта подгруппа образует один смежньй класс по $\Gamma\left(H_{e}\right)$.

Если $\varepsilon_{22}=0$, то $e-$ нулевая матрица и подгруппа $H_{e}$ одноэлементна. Пусть $\varepsilon_{22}=1$. Возьмем произвольную матрицу $b=\left(\beta_{i j}\right) \in H_{e}$. Вычисляя произведение $e b=b$, заключаем, что $\beta_{11}=\beta_{33}=0$,

$$
\begin{aligned}
& \beta_{12}=\varepsilon_{12} \beta_{22}, \\
& \beta_{13}=\varepsilon_{12} \beta_{23} .
\end{aligned}
$$

Отметим, что применяя (3) к случаю $b=e$, получаем

$$
\varepsilon_{13}=\varepsilon_{12} \varepsilon_{23}
$$

Аналогично, вычисляя произведение $b e=b$, заключаем, что

$$
\beta_{23}=\varepsilon_{23} \beta_{22} \text {. }
$$


Подставляя (5) в (2) и учитывая $(4)$, получаем

$$
\beta_{13}=\varepsilon_{12} \varepsilon_{23} \beta_{22}=\varepsilon_{13} \beta_{22}
$$

Используя равенства (2), (5) и (6), мы можем представить матрицу $b$ в виде

$$
\begin{aligned}
b & =\beta_{12} e_{12}+\beta_{13} e_{13}+\beta_{22} e_{22}+\beta_{23} e_{23} \\
& =\beta_{22}\left(\varepsilon_{12} e_{12}+\varepsilon_{13} e_{13}+e_{22}+\varepsilon_{23} e_{23}\right)=\beta_{22} e .
\end{aligned}
$$

Если $c=\left(\gamma_{i j}\right)-$ другая матрица из $H_{e}$, то

$$
b c=\beta_{22} e \cdot \gamma_{22} e=\beta_{22} \gamma_{22} e=\gamma_{22} \beta_{22} e=\gamma_{22} e \cdot \beta_{22} e=c b
$$

Таким образом, группа $H_{e}$ абелева и потому совпадает со своим верхним гиперцентром.

\section{СПИСОК ЦИТИРОВАННОЙ ЛИТЕРАТУРЫ}

[1] Oates S., Powell M. B. Identical relations in finite groups // J. Algebra. 1964. V. 1. № 1. P. 11-39.

[2] Perkins P. Bases for equational theories of semigroups // J. Algebra. 1969. V. 11. № 2. P. 298-314.

[3] Шеврин Л.Н., Волков М. В. Тождества полугрупп // Изв. вузов. Матем. 1985. № 11. C. $3-47$.

[4] Volkov M. V. The finite basis problem for finite semigroups // Math. Japonica. 2001. V. 53. № 1. P. 171-199.

[5] Мальцев А.И. Алгебраические системы. М.: Наука, 1970.

[6] Сапир М.В. Проблемы бернсайдовского типа и конечная базируемость в многообразиях полугрупп // Изв. АН СССР. Сер. матем. 1987. Т. 51. № 2. С. 319-340.

[7] Клиффорд А., Престон Г. Алгебраическая теория полугрупп. Т. 1, 2. М.: Мир, 1972.

[8] Шеврин Л.Н.К теории эпигрупп. І // Матем. сб. 1994. Т. 185. № 8. С. 129-160.

[9] Сапир М.В. Существенно бесконечно базируемые конечные полугруппы // Матем. сб. 1987. T. 133. № 2. C. 154-166.

[10] Jackson M. Small inherently nonfinitely based finite semigroups // Semigroup Forum. (to appear). 